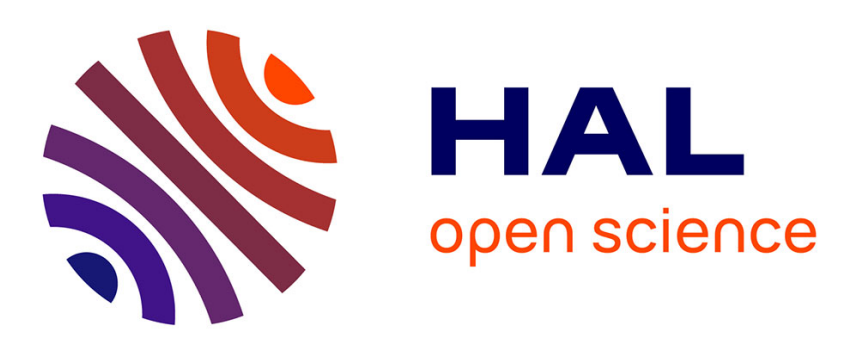

\title{
Optimal Stochastic Excitation for Linear Flaw Detection in a Solid Material
}

Nesrine Houhat, Sébastien Ménigot, Tarek Boutkedjirt, Redouane Drai, Jean-Marc Girault

\section{To cite this version:}

Nesrine Houhat, Sébastien Ménigot, Tarek Boutkedjirt, Redouane Drai, Jean-Marc Girault. Optimal Stochastic Excitation for Linear Flaw Detection in a Solid Material. 23rd Iberoamerican Congress on Pattern Recognition, Nov 2018, Madrid, Spain. 10.1007/978-3-030-13469-3_27 . hal-01982560

\section{HAL Id: hal-01982560 https://hal.science/hal-01982560}

Submitted on 15 Jan 2019

HAL is a multi-disciplinary open access archive for the deposit and dissemination of scientific research documents, whether they are published or not. The documents may come from teaching and research institutions in France or abroad, or from public or private research centers.
L'archive ouverte pluridisciplinaire HAL, est destinée au dépôt et à la diffusion de documents scientifiques de niveau recherche, publiés ou non, émanant des établissements d'enseignement et de recherche français ou étrangers, des laboratoires publics ou privés. 


\title{
Optimal Stochastic Excitation for Linear Flaw Detection in a Solid Material
}

\author{
Nesrine Houhat ${ }^{1}$, Sébastien Ménigot ${ }^{2,3}$, Tarek Boutkedjirt ${ }^{4}$, Redouane Drai ${ }^{1}$, \\ and Jean-Marc Girault ${ }^{2,3}$ \\ 1 Research Center in Industrial Technologies CRTI, P.O. Box 64, Cheraga 16014, \\ Algiers, Algeria. n.houhat@crti.dz, r.drai@crti.dz \\ 2 Eseo Group, Angers, France. sebastien.menigot@eseo.fr, \\ jean-marc.girault@eseo.fr \\ 3 LAUM, CNRS UMR 6613, Le Mans Iniversité, Le Mans, France. \\ 4 Physics of Ultrasound Reseach Team, Faculty of Physics, USTHB, Algiers, Algeria. \\ tboutkedjirt@usthb.dz
}

\begin{abstract}
The field of ultrasonic nondestructive testing has known a great development during the recent years. In order to increase the flaw detection sensitivity, many improvements have been made in the equipment and the sensors technology. In the present work, the optimal command which maximizes the flaw detection is investigated experimentally. A parametric optimization consisting of finding the optimal excitation frequency which maximizes the Euclidean distance between a reference medium and a medium with a linear flaw has been obtained automatically by using the gradient descent algorithm. Moreover, the waveform excitation optimization has been considered. A set of stochastic signals have been transmitted to the medium. A closed loop optimization process based on a genetic algorithm allowed to find the optimal excitation without a priori knowledge on the shape of the signal. This optimal excitation converged to a sinusoidal pulse with the optimal frequency found by the parametric optimization.
\end{abstract}

Keywords: Optimal command · Nondestructive testing · Gradient descent algorithm · Genetic algorithm · Ultrasound

\section{Introduction}

The ultrasound nondestructive testing (NDT) domain has known a great development during the last decade, especially by the advent of nonlinear NDT which permits the detection of microscopic damages such as cracks. Nevertheless, the introduction of the optimal command in such systems remains quite new and little-used. Fink has proposed a time reversal closed loop system which makes possible the optimization of the signal to noise ratio (SNR) for a linear behavior system [1]. Precursory works using the optimal command principle for nonlinear systems were initiated in $[2-5]$ in the field of medical contrast imaging. The originality of this work was to search for the optimal input excitation 
parameter/shape without any a priori knowledge of the studied system, by using well known optimization algorithms. However, the definition of an efficient cost function enabling to express correctly the optimization purpose remains the main difficulty. The stochastic approach is principally devoted to be used for nonlinear systems. Indeed, it allows to find the stochastic optimal command which improves the simple frequency optimization results. The present study is an extension to the NDT domain, of previous works $[4,5]$, in which the optimal command was applied in ultrasound medical imaging for the improvement of the image contrast. It is a preliminary work which consists in setting up an experimental closed loop platform allowing to experiment the procedure described in [4]. It constitutes a first step in the validation of the stochastic optimization approach for linear systems since our ultimate goal remains its application on nonlinear systems. The optimal excitation wave enables us the best discrimination between two media: a reference one without flaw and a medium with a linear flaw. An efficient cost function able to quantify the result of the optimization process will be defined by the Euclidean distance between the echoes emanating from the two media. A conventional ultrasound measuring system is therefore changed into a new system including a feedback. First, a parametric optimization is achieved. The parameter to be optimized is the transmitted frequency by using a gradient descent algorithm [6]. This first result constitutes a suboptimal solution. For an optimal solution, the excitation waveform optimization is considered. A set of stochastic signals is then transmitted to the investigated media and the optimal excitation can be found automatically by a genetic optimization algorithm [7]. The results obtained from the parametric optimization and the shape optimization will be compared.

\section{Method and material}

\subsection{Closed loop system for linear flaw detection}

The principle of our method is to add a feedback to a conventional open loop ultrasound system for linear flaw detection (Fig.1). For an iteration $k$, an excitation signal $x_{k}(t)$ is transmitted to the reference medium (switch position 1 ), then, to the medium with the linear flaw (switch position 2). The temporal received signal indicates the existence of a flaw by the presence of an echo positioned between the excitation and the medium back face echo. Adding a feedback to such a system permits us to optimize the flaw detection thanks to the input excitation without a priori information about the transducer and the medium. An Euclidean distance $d_{k}$ is computed between the two media. An optimization algorithm is used to find the optimal command which discriminates to the best the two media by maximizing the Euclidean distance $d_{k+1}$. For the parametric optimization, $x_{k}(t)$ is a Gaussian modulated sinusoidal pulse with a frequency $f_{k}$ and a duration of $1 \mu \mathrm{s}$. The power of the transmitted signal $E_{x}$ is adjusted to the power of a reference one $E_{x_{r e f}}$ with a transmitted frequency of $1 \mathrm{MHz}$ and with an amplitude of $A_{0}$. The amplitude of the transmitted pulse is then given by: 


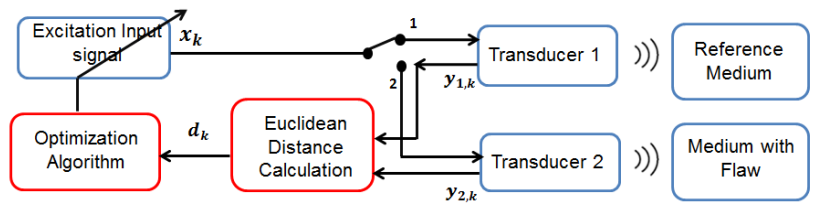

Fig. 1. Bloc diagram of the optimization closed loop system.

$$
A=\sqrt{\frac{A_{0}^{2} E_{x_{r e f}}}{E_{x}}}
$$

\subsection{Cost function}

Our main goal through this study is the improvement of the flaw detection. For this, we compare two media, a reference medium without flaw and a medium with the same characteristics including a linear flaw by considering an Euclidean distance between them. The greater the distance is, the more the flaw is highlighted. The cost function to be maximized, here, is an Euclidean distance between the temporal signal power received from the reference medium and those received from the medium with a flaw. The optimization of the Euclidean distance between the two media amounts to maximize the power of the flaw echo:

$$
d_{k}=\sqrt{\left(E_{1, k}-E_{2, k}\right)^{2}},
$$

where $E_{1, k}$ is the power of the temporal signal emanating from the medium with flaw and $E_{2, k}$ is the power of the temporal signal received from the reference medium.

\section{$2.3 \quad$ Algorithms}

Gradient Descent Algorithm For the parametric optimization, a gradient descent algorithm is used to find the suboptimal command. This algorithm has been chosen because the expression of the Euclidean distance as a function of frequency is unknown. The optimization is based on the selection of the best transmitted frequency $f^{*}$ which maximizes the Euclidean distance between the two media $d_{k}[6]$ :

$$
f^{*}=\arg \max _{f}(d(f))
$$

The gradient descent is an iterative algorithm defined by the recurrence relation:

$$
f_{k+1}=f_{k}+\mu_{k}\left(\nabla d\left(f_{k}\right)\right)
$$


where $f_{k}$ is the transmitted frequency at the iteration $k$. The coefficient $\mu_{k}$ governs the speed of convergence and $\nabla d\left(f_{k}\right)$ is the Euclidean distance gradient at the iteration $k$ for the transmitted frequency $f_{k}$ and is given by the expression:

$$
\nabla d\left(f_{k}\right)=\frac{\partial d}{\partial f} \approx \frac{d_{k-1}-d_{k}}{f_{k-1}-f_{k}} .
$$

The coefficient $\mu_{k}$ sets the speed of convergence as follows:

$$
\mu_{k}= \begin{cases}0 & \text { if } k \leq 3 \\ \Delta f & \text { if } k=4 \\ \mu_{k-1} & \text { if } \operatorname{sgn}\left[\nabla\left(d\left(f_{k}\right)\right)\right]=\operatorname{sgn}\left[\nabla\left(d\left(f_{k-1}\right)\right)\right] \\ -\frac{\mu_{k-1}}{2} & \text { if } \operatorname{sgn}\left[\nabla\left(d\left(f_{k}\right)\right)\right] \neq \operatorname{sgn}\left[\nabla\left(d\left(f_{k-1}\right)\right)\right]\end{cases}
$$

where $\Delta f$ was fixed to $1 \mathrm{MHz}$. This value must be chosen in order to ensure a good compromise between the speed of convergence and the robustness. The sign function $\operatorname{sgn}(t)=1$ for $t>0,0$ for $t=0$ and -1 for $t<0$. For $k=\{1,2,3\}$, the first three frequencies must be given initially. These three values can have an influence on the speed of convergence of the algorithm under the concavity hypothesis of the algorithm.

Genetic Algorithm For the shape optimization, the search of the optimal command $w_{k}^{*}$ is based on the investigation of stochastic signal which maximizes the Euclidean distance by using the genetic algorithm [7] that is $w_{k}^{*}=$ $\arg \max _{w_{k}}\left(d\left(f_{k}\right)\right)$. At the first generation $(k=0), 12$ stochastic signals are randomly chosen from a continuous uniform distribution. They constitute the initial population for the genetic optimization process. Each signal $x_{k}=A . w_{k}$ is composed from 40 samples with a time duration of $1 \mu \mathrm{s}$. In order to compare results obtained from the parametric optimization, the transmitted stochastic signal amplitude $\mathrm{A}$ is adjusted according to equation (1). For the next generation $k+1$, the 6 best signals maximizing the cost function are kept to become parents. 6 new stochastic signals named offspring are generated by the crossover operator which mixes the best parent with one of the 5 remaining parents. A rate of $40 \%$ of the samples corresponding to the mutation rate are mutated to obtain a robust optimization. It should be noted that these settings could be reached from several tests.

\section{Experimental study}

\subsection{Samples description}

Two aluminium cylindrical blocs with identical dimensions $80 \mathrm{~mm} \times 80 \mathrm{~mm}$ were considered (Fig. 2). A hole of $4 \mathrm{~mm}$ diameter and $20 \mathrm{~mm}$ depth was drilled on the back side of the first sample in order to simulate a linear flaw. 


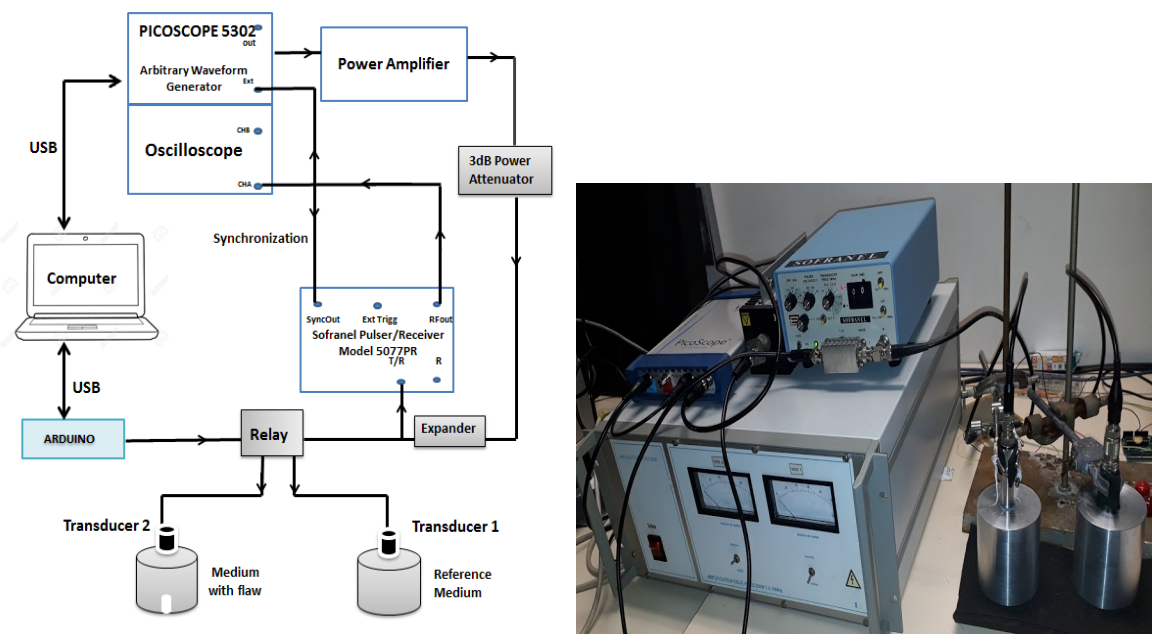

Fig. 2. Experimental setup.

\subsection{Experimental setup}

The experimental setup is described in Fig.2. The excitation signal $x_{k}(t)$ was generated digitally by Matlab (Mathworks, Natick, MA, USA) with a computer. It was transmitted to a PicoScope PS5203 arbitrary waveform generator (PicoTech, St Neots, UK) via USB, then amplified by $50 \mathrm{~dB}$ using a power amplifier (AAP-200-1-10, Adece, Veigné, France) which was protected by a $3 \mathrm{~dB}$ high power attenuator (HFP-5100-3/3-NM/ NF, Trilithic, Indianapolis, IN, USA). The signal was transmitted to the reference medium from a $2.25 \mathrm{MHz}$ PZT single element transducer (IM-2.25-12-P, Imasonic Sas, France) with a fractional bandwidth of $72 \%$ at $-6 \mathrm{~dB}$, then, to the medium with flaw from a paired transducer. This switching was possible by a relay circuit (TQ2-5V) commanded by the computer via an Arduino Uno micro controller. The received wave from the medium was collected by the same transducer (echo mode) and transmitted to a Sofranel Squarewave Pulser/Receiver Model 5077PR (Olympus NDT Inc, Waltham, USA) via a diode bridge. For each iteration, ten acquisitions were made by an oscilloscope (PicoScope PS5203) to increase the SNR. Finally an averaged echo was transmitted and recorded by the computer.

\subsection{Results}

Parametric optimization The first step of the study a so called "empirical optimization" was to check for the existence of a global maximum of the Euclidean distance versus frequency. For this purpose, a frequency sweeping in a range between $1 \mathrm{MHz}$ and $6 \mathrm{MHz}$ with a step of $50 \mathrm{kHz}$ was achieved. The obtained result is represented in Fig.3 (red solid line). This first result indicates that the cost function has a global maximum localized at about $f=3.3 \mathrm{MHz}$. This justifies the use of the gradient descent algorithm which operates under the 
convexity hypothesis of the cost function. This maximum can also be reached automatically with the gradient descent algorithm. The results of the first fifty iterations are shown in Fig.3 (black solid line). We can note the convergence of the distance to the maximum, and that the values obtained automatically are very close to those obtained empirically by the first experiment. It can also be noticed for the gradient optimization, a little variation of the cost function value for the same frequency. This observation is directly due to the experimental conditions.

Fig.4 shows the results of the gradient descent algorithm optimization. Variations of the distance (Fig.4.a) and the transmit frequency (Fig.4.b) versus iterations for two different initializations $\left(f_{1}=1 \mathrm{MHz}, f_{2}=1.2 \mathrm{MHz}, f_{3}=1.3 \mathrm{MHz}\right.$ and $f_{1}=$ $\left.6 \mathrm{MHz}, f_{2}=5.8 \mathrm{MHz}, f_{3}=5.5 \mathrm{MHz}\right)$ are presented. For both cases, after 30 iterations, the transmit frequency converges to the same value $f=3.2 \mathrm{MHz}$ while the distance value shows weak fluctuations around an average value of 40 a.u (arbitrary unit). These fluctuations are probably due to the experimental conditions. The optimal transmit frequency can be reached automatically for the two algorithm initializations with the same speed of convergence.

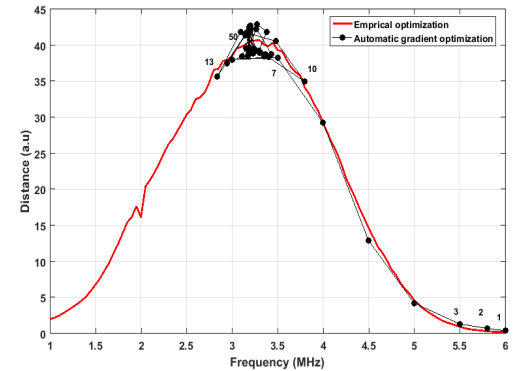

Fig. 3. Empirical optimization: Euclidean distance measurement for a transmit frequency of 1 to $6 \mathrm{MHz}$ with a step of $50 \mathrm{kHz}$ (red solid line) and the gradient descent automatic optimization (black line)for the 50 fifty first iterations.
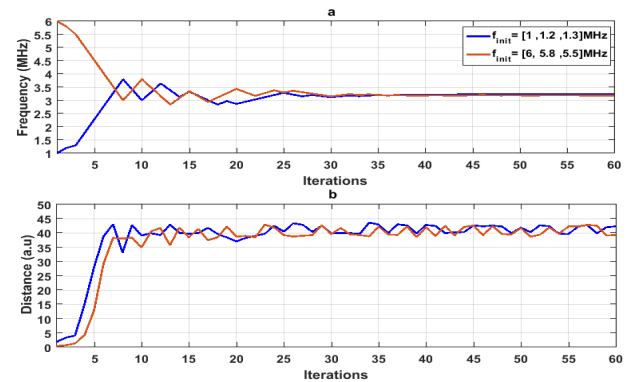

Fig. 4. Gradient descent algorithm optimization results for two different initializations: $f_{1}=1 \mathrm{MHz}, f_{2}=1.2 \mathrm{MHz}, f_{3}=1,3 \mathrm{MHz}$ and $f_{1}=6 \mathrm{MHz}, f_{2}=5.8 \mathrm{MHz}, f_{3}=5.6 \mathrm{MHz}$. (a) Transmitted frequency versus iterations. (b) Euclidean distance versus iterations.

Shape optimization Fig.5.a shows the distance as a function of generations $k$ (solid line) and the parametric optimization result (dashed line) obtained previously. We can note that the cost function has reached an optimal value of 44 a.u after 430 generations. This value is almost identical to those obtained with the optimal transmitted frequency which was around 41 a.u. Fig.5.b represents the corresponding optimal stochastic command $w_{k}^{*}$. For the comparison of the results obtained by both parametric and shape optimization, an illustration of the optimal stochastic signal and the sinusoidal pulse at the optimal frequency is 

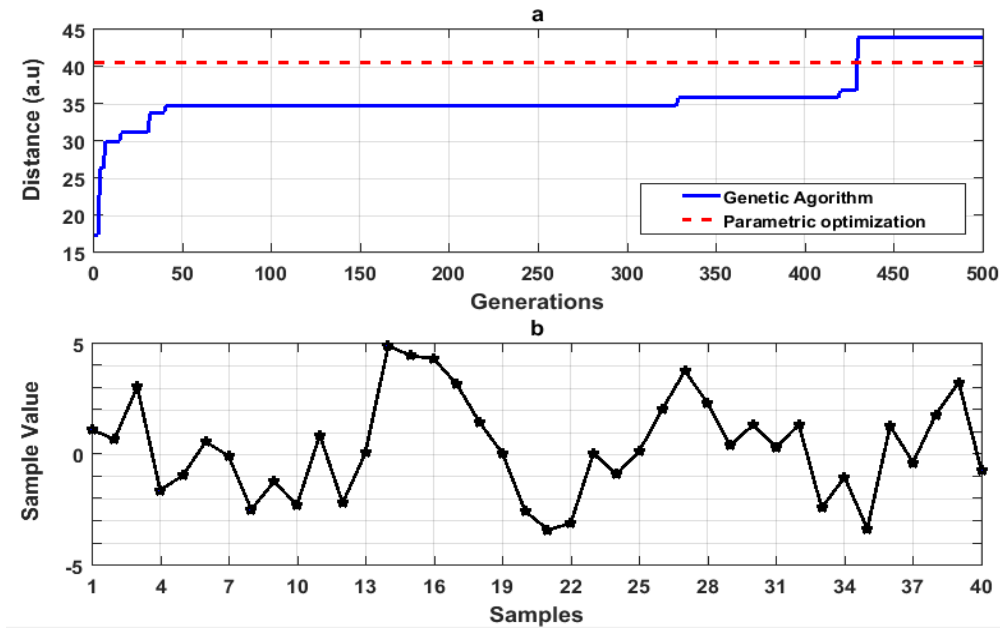

Fig. 5. a) Genetic algorithm optimization of the Euclidean distance versus generations (blue solid line) and the parametric optimization result in dashed line, b) Optimal transmitted stochastic signal .
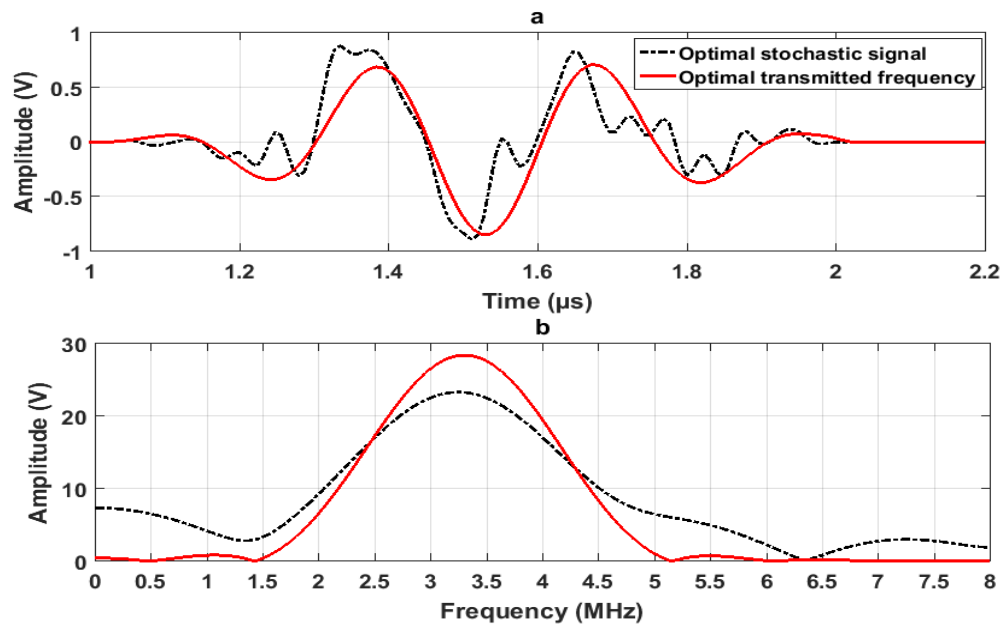

Fig. 6. Optimal stochastic signal (dashed line) and the sinusoidal pulse at the optimal transmitted frequency (red solid line) and their respective spectra.

given in Fig.6.a. Their respective spectra are shown in Fig.6.b. The two signals have been resampled to have the same sampling frequency of the experimental system and filtered with the same Hann window, then, rescaled in order to have the same power. The optimal stochastic signal converges to a sinusoidal pulse at the optimal frequency. We suppose that a better matching between the two results could have been reached for a greater number of generations. It can be said that for a linear flaw type, the use of stochastic signals for the shape op- 
timization reinforces the result of the sine pulse parametric optimization. The expected optimal excitation, here, is the time reversed impulse response of the system including the transducer and the medium.

\section{Conclusion}

In the present study, the best excitation wave which enabled to maximize the linear flaw detection was investigated through two process types, parametric and shape optimization. An experimental closed loop system was set up to find automatically the suboptimal/ optimal command. The cost function considered was an Euclidean distance between two media: a reference one and a medium with a linear flaw. The main outcome was the performance similarity between the parametric and the stochastic approaches. Nevertheless, the stochastic approach didn't require any a priori knowledge nor initialization values. For a linear flaw type, the optimal stochastic command tends to the time reversal solution which is a sinusoidal pulse at a fixed frequency. The stochastic approach may be more efficient for nonlinear systems, even for cases where the cost function is no longer convex, for which the use of simple parametric optimization is compromised. This work is ongoing and will be presented in a future paper.

\section{Acknowledgment}

The authors thank Dr Jean- Marc GREGOIRE (Université Franois Rabelais, Inserm, Imagerie et Cerveau, UMR U930, France, Tours) for his realizations, helpful discussions and advices about the experimental setup.

\section{References}

1. M. Fink,: Time reversal of ultrasonic fields. I. Basic principles" IEEE Trans. on Ultraso. Ferroelectr. Freq Contr. 39(5), 555-566 (1992).

2. S. Ménigot, J. M. Girault, I.Voicu, and A. Novell,: Optimization of contrast-to-tissue ratio by frequency adaptation in pulse inversion imaging. IEEE Trans. on Ultrason. Ferroelectr. Freq. Contr., 59(11), 2431-2438(2012).

3. S. Ménigot, and J. M Girault,: Optimization of contrast resolution by genetic algorithm in ultrasound tissue harmonic imaging. Ultrasonics, 71, 231-244 (2016).

4. J. M Girault, and S. Ménigot,: Contrast optimization by metaheuristic for inclusion detection in nonlinear ultrasound imaging. Physics Procedia 70, 614-617 (2015).

5. S. Ménigot, M. Geryes, J. Charara, and J. M. Girault,: Inclusion/Flaw detection in ultrasound imaging through optimization of random transmitted wave. In: Acoustics 2013, New Delhi, (2013).

6. B. Widrow, and S. Stearns, Adaptive Signal Processing, Prentice Hall, Englewood Cliffs, USA, 1985.

7. R.L. Haupt, S.E. Haupt: Practical Genetic Algorithms, Second ed., John Wiley and Sons Inc, Hoboken, NJ, USA (2004). 